A. B. Meyer: Ueber einige Tauben von Borneo etc.

Das vorliegende Paar ist ein altes, sehr ausgefärbtes. Fs fehlt auf der Unterseite des $\delta$ jegliche Andeutung des gelben Tones von fulvicollis, es ist die düster grüne Farbe der Schwanzfedern etwas lebhafter grün und es sind die Hosen lebhafter gelb; sonst gleicht es fulvicollis.

Das Weibchen, welchem zum Vergleiche mir kein $q$ von fulvicollis vorliegt, hat den Oberkopf schieferfarbig, die Oberseite dunkel olivengrün, den Schwanz etwas lebhafter grün. Die Unterseite der Flügel ist dunkel schieferfarben, Kinn und Kehle lebhaft gelbgrün, Hals und Brust graugrün, Bauch heller, Brust- und Bauchseiten in's Blaugraue ziehend; untere Schwanzdecken grüngrau mit Weiss und hell Isabellfarbe breit gesäumt, sonst dem $\delta$ gleich; Long. al. 0,144 m, caud. 0,082 m, rostri (culm.) $0,014 \mathrm{~m}$, tarsi $0,017 \mathrm{~m}$.

Dresden, den 27. October 1890.

\title{
Verfahren zur Mumification \\ von Vögeln und anderen zoologischen objecten.*)
}

Von

Prof. Dr. Johannes Frenzel, in Cordoba.

Es ist bekannt, dass sich für wissenschaftliche Zwecke am besten eine dauernde Conservirung der Präparate in Spiritus eignet, nachdem sie unter Umständen vorher mit anderen $H a ̈ r$ tungs mitteln, wie Sublimat, Chromsäure etc. behandelt worden waren. So sehen wir namentlich Reptilien, Amphibien, Fische, Würmer und Coelenteraten in Spiritus aufbewahrt. Um vieles einfacher erscheint freilich die Conservirung von Insecten, bei denen das unmittelbare Trocknen genügt, während man bei der Behandlung von Vögeln schon etwas complicirter verfahren muss. Auch diese setzt man zuweilen in Spiritus, wobei man noch den Vortheil geniesst, Skelett und Weichtheile zu erhalten. Leider aber wirkt bekanntlich, von anderen Unbequemlichkeiten abgesehen, ein längeres Verweilen in dieser Flüssigkeit so nachtheilig auf das Gefieder, dessen Farben verblassen und stumpf werden, dass dadurch das hauptsächlichste Unterscheidungsmerkmal der Vögel

*) Siehe: Zoologischer Anzeiger 1888 No. 295. 
verloren gehen kann, in Folge dessen derartige Präparate für systematische Zwecke nicht recht brauchbar sind. Ausserdem bleibt ihre Handhabung immer eine unbequeme, zumal sich das Gefieder wie ein Schwamm mit Spiritus vollsaugt.

Dieser Umstände halber blieb daher immer noch die beste Manier, um Vögel für Museumszwecke zu benutzen, die, sie abzubalgen und den Balg an der Luft zu trocknen; und derartige B älge sind nun auch fast durchgehends völlig ausreichend für den eigentlich wissenschaftlichen Zweck, da es sich zunächst ja nur darum handelt, ihre Merkmale festzustellen, sie in das System einzureihen und zu klassificiren, und schliesslich als Belegstücke aufzubewahren. Lässt sich doch solch' ein Balg bequem handhaben und in verhältnissmässig kleinen Kästen aufbewahren, wodurch selbst uiber eine grössere Anzahl eine vollkommene Uebersichtlichkeit erzielt wird, was aber nicht mehr in dem Maasse der Fall bleibt, wenn der Balg ausgestopft und aufgestellt wird.

Die Nachtheile, welche die Herstellung derartiger wie uiberhaupt auch anderer Bälge mit sich bringt, liegen z. T. darin, dass dieselbe einige Schwierigkeit verursacht und zeitraubend ist, so dass sich nicht jeder, der es wohl gerne möchte, damit befassen kann, woraus folgt, dass das Sammeln immer ein beschränkteres bleibt. Sieht man davon ab, dass der Sammler im Abbalgen unterrichtet sein muss, so bedarf er bekanntlich auch noch einer gewissen Uebung und Gewandtheit, um nicht zuviel Zeit dabei zu opfern, denn wie oft hat nicht selbst ein geschickter Präparator so viel Material gesammelt, dass er das nicht zu bewältigende muss verderben lassen. Man bedenke fernerhin, dass nicht nur ein Ornithologe, sondern auch ein Geograph, Mineraloge oder Botaniker in der Lage sein wird, auf Reisen und Excursionen Vögel zu schiessen, ohne im Stande zu sein, sie zu conserviren. Ja, manchem Zoologen von Fach geht es darin nicht viel besser. Meistentheils hat ja der wissenschaftlich Reisende so viel verschiedene Aufgaben vor sich, dass er jeder derselben nur eine beschränkte Aufmerksamkeit zuwenden kann, und dies mag es wünschenswerth erscheinen lassen, eine möglichst vielfache, leichte und kurze Methode mitzutheilen, um besonders Vögel und kleinere Säugethiere, ferner aber auch andere Objecte, wie Panzerfische, Reptilien, Insecten, Krebse, Echinodermen etc. so zu präpariren, dass sie den gewöhnlich zu stellenden Anforderungen genügen. Auch die im Grossen betriebene, Jagd auf Schmuckvögel für in- 
dustrielle Zwecke dürfte davon einigen Gewinn ziehen. Besonders aber wird jeder davon Gebrauch machen können, der entweder nicht viel Zeit $\mathrm{zu}$ opfern oder aber keine hinreichende Uebung im Abbalgen hat, wie z. B. Aerzte, Förster, Jäger, Landleute etc+

Um daher alle diese in den Stand zu setzen, mehr, als dies bisher möglich war, zoologische Objecte zu sammeln und zu conserviren, möchte ich im Folgenden einige Mittel und Wege angeben, welche ich auch dem geübten Präparator empfehle, zumal wenn er grosse Massen von Material zu bewältigen hat. Das dabei anzuwendende Verfahren bezweckt, den Cadaver in seiner Ganzheit zu conserviren, wobei nöthigenfalls das Skelett für osteologische Zwecke erhalten bleibt und selbst die Eingeweide behufs einer genaueren anatomischen oder histologischen Untersuchung oder einer solchen nach Magen- und Darminhalt oder nach Eingeweidewürmern reservirt werden können. -

Um nun zu dem Verfahren selbst überzugehen, möchten wir an die einfachste Methode, um Thiere zu conserviren, anknüpfen, wie sie beim Insectensammeln ausgeïbt wird. Sie besteht bekanntlich im unmittelbaren Trocknen der Objecte, wenn das Klima nicht gar zu feucht ist. Unter sehr günstigen Umständen kann man dergestalt auch andere Objecte behandeln, wie etwa Krebse und Panzerfische, und es ist bekannt, dass selbst Kadaver von grossen Thieren und Menschen auf diese Weise mumificirt wurden. Es sei hier nur an den „heiligen Vogt von Sinzig“*) erinnert, dessen vollständig erhalten gebliebene Haut ibre natürliche Farbe fast ganz bewahrt haben soll und lose die steinhart gewordenen und zusammengeschrumpften Muskeln umschliesst. Da diese Mumie keinerlei Einschnitte etc. erkennen lässt, so nimmt man an, dass sie nur durch Austrocknung entstanden ist.

Derartige günstige Umstände sind im Allgemeinen aber nur selten auf dem Erdball anzutreffen. Grosse Trockenheit genügt allein nicht. Die Luft muss auch besonders rein von Fäulnissund Schimmelpilzen sein, und dann ist immer noch Gefahr vorhanden, dass vom Innern des Körpers aus die Zersetzung nach aussenhin fortschreitet, wenn ihr nicht durch eine sehr beschleunigte Austrocknung Einhalt geboten wird. Dies kann aber nun am allerwenigsten bei den Vögeln eintreffen, deren Federkleid die Austrocknung ungemein verhindert.

*) Siehe: „Die Gartenlaube“, herausgegeben von Ernst Keil. 1881, S. 248 ff.: „Der heilige Vogt von Sinzig“" von M. L. 
Ferner bringt eine mit Trockenheit verbundene Wärme, welche ja das Verdunsten sehr beschleunigt, meist noch recht ungünstige Umstände mit sich, nämlich den Insectenfrass, dem durch einfaches Trocknen hergestellte Präparate gar zu leicht anheimfallen. Durch eine kleine Aenderung kann aber diesem Uebel leicht abgeholfen werden, und wir gelangen zu einem zweiten, immer noch einfachen Präparationsverfahren, welches sich jedoch nur unter gewissen Umständen empfiehlt. Dasselbe bezieht sich auf eine kurze Bemerkung*), welche ich bei früherer Gelegenheit machte, wo es sich um das Trocknen von Echiniden handelte. Die Seeigel bieten nämlich, wenn man sie ohne Weiteres an der Luft absterben und trocknen lässt, ein recht trauriges Bild dar, indem die Stacheln ihre radiäre Stellung verlieren, umfallen und schief zu stehen kommen. Tödtet man diese Thiere aber in schwacher alkoholischer Sublimatlös ung, so wird dieser Uebelstand völlig vermieden und beim nachherigen Trocknen bleibt die natürliche Stellung der Stacheln erhalten. Zugleich wird dadurch ein wichtiger Vortheil errungen, welcher darin besteht, dass das Präparat an seiner ganzen Oberfläche derartig vergiftet ist, dass es vor Frass und Schimmelbildung genügend geschützt ist. Geradeso lassen sich nun zunächst auch andere Echinodermen, wie See- und Schlangensterne, behandeln, weiterhin dann noch mittelgrosse Krebse und allerlei Insecten, mit Ausnahme vielleicht solcher, deren zarte Behaarung etc. überhaupt keine Benetzung verträgt, wie Fliegen, Bienen etc., oder deren Farben zerstört werden, wie bei den grünen Flügeln von Heuschrecken. Wendet man bei behaarten Insecten aber starken Alkohol mit ca. $1 \%$ Sublimat an, indem man die Objecte einfach hineintaucht, um sie dann $\mathrm{rasch}$ zu trocknen, so vermeidet man leicht das Zusammenbacken der Härchen. Ebenso erreicht man bei Heuschrecken ein günstiges Resuitat, wenn man sie nach dem Tödten erst völlig trocken werden lässt und dann in Sublimatalkohol badet; dabei bleibt selbst die zarte grüne Farbe der Mantiden unverändert.

Im äussersten Nothfall können noch andere Objecte durch ein derartiges Sublimatbad conservirt werden, so etwa Panzerwelse und andere Fische mit wenig Fleisch oder harter Bedeckung,

*) Siehe: Zoologische Jahrbücher Bd. I Heft 1, 1886: „Verfahren zur Herstellung von Zoologischen und Anatomischen Präparaten mittelst der Glycerindurchtränkung. 
z. B. Seepferdchen und kleine Rochen, dann ferner kleine Vögel und Säuger, wie etwa Fledermäuse.

Die Art und Weisse dieser Conservirung, worüber weiter unten noch Genaueres erfolgt, ist so einfach, dass sie jedem Laien anvertraut werden kann. Ein schwüles, feuchtes Klima verbietet sie aber in den meisten Fällen. Für manches, wie Frösche, ist sie nur ein karger Nothbehelf, und mit Ausnahme der Insecten und Echinodermen liefert sie ïberall nur mangelhafte Resultate, was hauptsächlich daher kommt, dass im In n e rn der Thierkörper eine starke Fäulniss stattfindet, wenn nicht ein sehr rasches Austrocknen möglich ist. Krebse und ähnliche Objecte werden daher leicht missfarbig und Vögel verlieren ihre Federn.

Um daher diesem Uebel zu steuern, ist auch eine innere Desinfection erforderlich oder - in manchen Fällen - eine Entfernung der Eingeweide. Dies letztere allein ist nämlich, besonders bei Vögeln und Säugern, im Allgemeinen nicht genügend, obgleich im Innern des Thierkörpers eigentlich nur der Darmtractus als ein Heerd der Bacterienwucherung anzusehen ist. Aber als gefährliche Eingangsthore für Bacterienkeime müssen wir noch die natürlichen Leibesöffnungen, ferner Schusswunden und andere Verletzungen betrachten.

Alle diese Umstände legen uns daher eine weitergehende Vergiftung und Desinfection des Thierleibes nahe; erstere gerichtet gegen Insectenfrass, letztere gegen Fäulniss und Schimmel.

Eine solche Behandlung kann aber nur von Innen heraus stattfinden, da, wie bekannt, die Thierhaut sehr schwer für Flüssigkeiten durchlässig ist, weshalb ein einfaches Baden oder selbst ein längeres Verweilen in der Conservirungsflüssigkeit obigen Zweck nicht erreichen lässt.

Das nunmehr anzuwendende Verfahren beginnt damit, dass man den gefährlichsten Fäulnissheerd, den Darmtractus beseitigt. Um die Federn der Vögel dabei nicht zu beschmutzen, befeuchtet man sie am After mit Spiritus und streicht sie zur Seite. Sodann schiebe man einen hakenförmig gebogenen Draht in den After und ziehe nun vorsichtig den Darm heraus, der gewöhnlich am Magen abreisst. Dieser kann jedoch ohne Gefahr im Leibe bleiben, während der Darm entweder sogleich frisch auf seinen Inhalt, auf Eingeweidewürmer etc. untersucht, oder in Spiritus conservirt wird, am besten in derselben Flüssigkeit, welche weiter unten zum äusseren Vergiften dient. Bei kleineren Objecten ist aber das 
Entfernen des Darmes gar nicht einmal nöthig, z. B. bei Colibris, und hier genügt es, mit einem kleinen Draht oder einer Nadel die Baucheingeweide vom After aus zu durchstossen, damit die später anzuwendende Injectionsfluissigkeit gut eindringen kann.

Ein ähnliches Durchstechen ist ausser dem Aushaken übrigens auch bei grösseren Tieren zu empfehlen, indem man den spitzen Draht vom After aus in die Brusthöhle, in das Brustfleisch und in die Schenkel stösst.

Da es nach äusseren Merkmalen bekanntlich oft schwer hält, das Geschlecht eines Thieres zu erkennen, was namentlich für die Vögel gilt, so ziehe man gleichfalls mit dem Aushaker oder mit einer passenden Pincette ein Stück des Hodens oder des Eierstockes heraus. $\mathrm{Zu}$ günstiger Jahreszeit wird der Geübte schon mit blossem Auge dieses Stïck erkennen. Den schnellsten und sichersten Aufschluss giebt in allen Fällen das Mikroskop.

In sehr vielen Fällen wird man nun ein Interesse daran haben, den Darmtractus oder den Geschlechtsapparat möglichst intact zu erhalten und diese Organe für spätere anatomisch-histologische Zwecke autbewabren. Hierzu empfiehlt es sich, anstatt durch den After auszuhaken, mit einem Längsschnitt die Bauchwand zu öffnen, wobei man vorher ebenfalls die mit Spiritus angefeuchteten Federn bei Seite streicht. Man kann nun mit Bequemlichkeit die gewünschten Organe herausnehmen, worauf man den leeren Raum mit Watte, Wolle oder dergl. vollstopft und sodann die Bauchhaut mit einigen Stichen wieder zunäht, eine Verrichtung, welche nicht die geringste Uebung erfordert und sich schnell ausführen lässt. Dies Verfahren ist nicht viel complicirter als das Aushaken, gewährt aber den bedeutenden Vortheil, dass man das Injiciren spart - von dem weiter unten noch zu sprechen ist - da durch die Nahtlücken genügend viel Conservirungsflüssigkeit in den Körper eindringen kann. Ich möchte daher diese Art der Präparation ganz besonders dem wissenschaftlich Reisenden oder dem Fachzoologen empfehlen, welcher das Abbalgen als zu zeitraubend empfindet und welcher gleichzeitig die gesammelten Okjecte möglichst vielseitig verwerten will.

Wenn man den Cadaver ganz intact gelassen oder die Eingeweide nur ausgehakt hat, was sich besonders empfiehlt, wenn man das darauf folgende Bad aus irgend einem Grunde gänzlich vermeiden will, so hat nunmehr die Injection zu erfolgen. Auch diese geschieht hauptsächlich durch den After, dann noch, 
wenn es Noth thut, durch den Rachen. Die dazu nöthige Flïssigkeit besteht am besten aus etwa 90 procentigem Alcohol (Spiritus vini), der am besten mit $Q$ uecksilbersublimat gesättigt ist. Im Nothfall genügt aber auch eine entsprechende Lösung eines anderen Desinficiens, wie Salicylsäure, Carbolsäure, Borsäure oder dergl.; doch ist Sublimat unter allen Umständen vorzuziehen, weil es am sichersten und energischsten wirkt.

Man braucht nur geringe Mengen einer derartigen Flïssigkeit. Das Einspritzen erfolgt mittelst einer gewöhnlichen kleinen Glasspritze, und man achte nur darauf, dass der Stempel derselben nicht aus Leder bestehe, da dieses durch die Einwirkung des Sublimates sehr schnell hart wird. Am besten eignet sich für einen derartigen Zweck die gewöhnliche Fadenumwicklung. Sehr gute Dienste leistet übrigens auch eine Spritzflasche, und im Nothfall reicht ein kleiner Glastrichter aus, in welchen man die Conservirungsflüssigkeit giesst.

Eine einmalige kräftige Einspritzung mit der Sublimatlösung reicht völlig zur inneren Vergiftung aus. Bei Thieren von Finkenoder Mäusegrösse genügen eine oder zwei Spritzen voll. Sehr kleine Objecte, wie Colibris, bedürfen kaum einer solchen Injection, da sie zumeist schnell genug trocknen; denn ihre Oberfläche ist im Verhältniss zu ihrem Volumen so gross, dass die Verdunstung des Wassers schneller von statten geht, als bei grösseren Objecten. Will man aber schön aussehende Präparate erzielen, so muss das Austrocknen recht langsam vor sich gehen, aus welchem Grunde sich dann wieder die Injection empfiehlt, damit während dem keine Fäulniss eintrete.

Ist nun aber die Bauchhöhle in der oben angegebenen Weise geöffnet worden, so fällt die Injection fort, und man kann sogleich zum äusseren Bade schreiten, dessen Zweck hauptsächlich darin besteht, die äussere Bedeckung des Präparates gegen Insectenschaden zu sichern. Wenn man bedenkt, wie oft der Sammler unter dieser Gefahr leidet und wie selbst die Museen fort und fort damit zu kämpfen haben, so kann ich nicht warm genug die Vergiftung durch Sublimat empfehlen, die auch auf abgezogene Bälge anzuwenden ist, wie wir noch sehen werden.

$\mathrm{Zu}$ dem Bade dient eine Flüssigkeit, die am besten aus $65-$ bis $70 \%$ igem Alcohol besteht; doch genügt auch der gewöhnliche Brennspiritus oder ein starker nicht zu sehr gezuckerter Schnaps. Darin ist etwa 1 bis $2 \%$ Sublimat gelöst, oder allen- 
falls ein anderes starkes Gift. Auch hierbei verdient aber das Sublimat den entschiedensten Vorzug. Es ist bequemer zu handhaben als z. B. Arsenikpräparate und bewirkt gleichzeitig einen sicheren Schutz gegen thierische wie auch gegen pflanzliche Feinde, gegen Mäuse und Insecten wie gegen Schimmel und Fäulniss. Es ist allerdings richtig, dass das Sublimat sich mit der Zeit verflüchtigt. Dies geschieht aber auch mit Arsenik oder Carbolsäure. Die Lösung von 1 bis $2 \%$ ist ferner so kräftig, dass selbst nach langen Jahren genügend davon zurückbleibt, um allen Angriffen zu widerstehen. Auch die Botaniker vergiften bekanntlich die Pflanzen ibrer Herbarien allgemein mit Sublimat.

Es ist zweckmässig, wenn man den anzuwendenden Sublimatspiritus noch mit Ka li-A la u n sättigt. Die so bereitete Flüssigkeit, von der man einige Liter haben muss, hält man am besten in einem grösseren Glase, einer zugedeckten Schüssel, einem gutschliessenden Tönnchen oder dergl., so dass man die Präparate hineinlegen kann. Blechgefässe eignen sich natürlich nicht. Für sehr grosse oder sehr zahlreiche Objecte empfehle ich, eine Holzkiste zu nehmen, welche man vorher innen mit heissem, fast kochendem Paraffin ausgegossen hat. Wenn das heisse Paraffin, dessen Temperatur ca. 110 bis $120^{\circ} \mathrm{C}$. sei, nämlich nur oberflächlich in das Holz eingedrungen ist, so macht es dies undurchlässig für Alcohol und man hat nur noch nöthig, die Kanten und Ecken der Kiste sorgfältig auszugiessen.

Der Vogelcadaver wird nun in dieser Flüssigkeit gebadet und bleibt am besten einige bis vierundzwanzig Stunden darin. Für kleine und frisch getödtete Objecte reicht schon ein starkes Benetzen des Gefieders aus. Angefaulte Präparate hingegen bedürfen einer längeren Behandlung. Der Metallglanz, den das Gefieder vieler Vögel, wie das der Colibris, mancher Enten etc. besitzt, leidet nicht im Mindesten durch den Alcohol, wohl aber durch Benetzung mit Wasser. Auch die zarten und leuchtenden Farben des Gefieders leiden kaum, und nach den zahlreichen Versuchen, welche ich bisher Gelegenheit hatte anzustellen, war sogar nach 24 stïndigem Verweilen im Bade keine Veränderung der Farben eingetreten. Man hüte sich aber davor, den Alcobol stärker, als angegeben, anzuwenden, da er sonst leicht dem Gefieder zu viel Fett entzieht. Eher kann man, wenn die Flüssigkeit schon wiederholt gebraucht ist, etwas starken Alcohol hinzugiessen, damit sie nicht zu schwach wird.

Cab. Journ. f. Ornith. XXXIV. Jahrg. No, 193. Januar 1891. 
Für besonders grosse Objecte wird es oft an einem passenden Gefäss oder an genügender Menge von Flüssigkeit fehlen. In solchen Fällen hilft man sich dadurch, dass man zunächst das Gefieder durch Begiessen durchnässt und hierauf den Cadaver in benetzte Tücher einwickelt. Man versäume dabei nui nicht, die innere Vergiftung recht sorgfältig vorzunehmen.

Durch die nunmehr angegebene Behandlung sollen folgende drei Zwecke erfüllt sein. Es ist erstens die Substanz des Cadavers so weitgehend wie möglich zu desinficiren, was in erster Linie durch die Injection erstrebt wird, zweitens wird die Haut durch das dem Sublimatalcohol zugefügte Alaun derartig g e g e r bt, dass Federn und Haare bei der weiteren Behandlung festhaftend bleiben, und drittens wird durch das Bad das Gefieder resp. der Pelz derartig vergiftet, dass die Präparate vor Insectenfrass oder dergl. sicher geschützt sind.

Wo letzteres Uebel nicht allzusehr zu befürchten ist, oder wo sonst welche Umstände das Baden in obiger Flüssigkeit nicht zulassen, gentigt oft schon die innere Behandlung, während a us s e n ein tüchtiges Einpulvern mit vergifteten Substanzen, wie etwa Gypsmehl, Insectenpulver oder dergl. plus Sublimat und der darauf folgende Trockenprocess, von dem jetzt zu sprechen sein wird, genügen.

Dieser Trockenprocess hat uns jetzt zu beschäftigen. Sobald nämlich die feuchte Behandlung vollendet ist, hebt man das Präparat aus der Flüssigkeit, drückt es mit den Händen aus, lässt es abtropfen und schlägt es in ein trocknes Tuch, in Löschpapier oder in sonstiges nicht geleimtes Papier. So eingewickelt kann es eine, mehrere bis 24 Stunden liegen. Zur Schonung des Glanzes und der Farben sei man jedoch flinker und hänge den schon ziemlich trocken gewordenen Cadaver, an den Beinen kopfabwärts, an einem luftigen, schattigen Ort zum weiteren oberflächlichen Trocknen auf, wozu einige Stunden zumeist genügen, da man nur so lange zu warten hat, bis das Gefieder trocken und locker ist. Dann kann schon an das Verpacken gegangen werden, und nur, wenn die Gegenden am After, Schnabel, an den Augen etc. noch Feuchtigkeit abgeben, so streue man sie mit Sand, Gypsmehl, Erde oder ähnlichem ein. Man glätte und ordne das Gefieder ein wenig, umwickle das Präparat mit Zeugläppchen oder Watte, und schiebe kleine Bäusche davon zwischen Flügel und Körper. Hierauf kann das Präparat endgültig in mehrere Lagen Löschpapier, Zeitungs- 
blätter ete. verpackt und ohne weitere Umstände und Zeitverlust an seinen Bestimmungsort transportirt werden. Hier kann man diese Mumien, da sie zumeist gedrückt und unansehnlich sein werden, wieder aufarbeiten resp. abbalgen, zu welchem Zweck man sie auf feuchtem Sande aufweicht, was sich ohne Muihe bewerkstelligen lässt.

Wenn man es mit dem Verpacken nicht allzu eilig hat und in grösserer Ruhe arbeiten kann, so ist man auch im Stande, von vorne herein schöne Präparate zu erzielen. In diesem Falle lässt man die gebadeten Objecte länger hängen und achtet darauf, dass das Gefieder schön aufgeht. Ist es windstill, so helfe man durch Schwencken und Blasen nach, oder pulvere die Federn mit trockenem Sand, oder einem Gemisch von solchem mit Gypsmehl ein und lasse das Präparat etwa eine Woche lang frei hängen, bis die Läufe oder die Fliigel anfangen steif zu werden. Hierauf lege man den Cadaver horizontal in die Dauerlage und belasse ihn ruhig in dieser Stellung bis er steif getrocknet ist. Da die während des Hängens nicht verdunstete Körperflüssigkeit nach dem Kopf zu gesunken ist, so wird dieser und die Halspartien noch weich sein, während die Extremitäten schon steifer sind. Eine Veränderung in die horizontale Lage ist daher des gleichmässigeren Trocknens wegen erforderlich. Gleichzeitig kann man jetzt dem Präparat die gewünschte Stellung geben.

Sollen nämlich die Objecte dauernd als „Mumien" conservirt werden, was mir durchaus nicht unzweckmässig erscheint, so möchte ich folgende Lage besonders anrathen: Man lege den Vogel auf die eine Seite und ziehe den entsprechenden Flügel nach vorne vom Körper ab, so dass er der Unterlage aufliegt, auf welcher er mit einigen Nadeln oder dergl. festgehalten wird. Der andere Fliigel bleibt in der Normal-Ruhelage am Körper, und den Kopf kann man durch einen Wattebausch stützen. Auf diese Weise gewinnt man mit einem Blick eine Uebersicht über das ganze Thier, schneller, als wenli es auf dem Rücken liegt, denn man sieht sowohl die Ober- wie die Unterseite der Flügel, sowohl Rücken wie Bauchseite. Auch die Schwanzfedern können etwas gespreizt werden, wobei man sie, bis die Haut trocken genug ist, durch Nadeln, Papierstreifen u. s. w. unterstiitzt.

Wenn dieser Trockenprocess langsam vor sich geht, so verdunsten die Körperflüssigkeiten so allmählich, dass nur ein geringeres Schrumpfen der Weichtheile stattfindet. Vor Allem die 
Fleischsubstanz bleibt mehr schwammig und behält ihr Volumen annähernd bei. Die Hauptsache dabei ist, wie gesagt, das langs a me Trocknen, das man durch Bedecken mit Papier, mit einem Kasten oder derartigem nach Belieben reguliren kann. Um dem Präparat noch ein volleres Aussehen zu geben, kann man, nachdem die Eingeweide ausgehakt oder durch den Bauchschnitt entfernt worden sind, die Bauchhöhle, den Hals und sogar die Brustmusculatur vom After, von der Bauchöffnung und vom Rachen aus mit etwas Watte oder dergl. nachstopfen.

Die nach diesem Verfahren hergestellten Vogelmumien erinnern an das Aussehen frisch geschossener Vögel, abgesehen davon, dass sie steif sind. Sie werden daher auch einigen ästhetischen Anforderungen Rechnung tragen, so dass man jede derartige Mumie als Museumsexemplar verwenden kann. Jedenfalls aber ist dieselbe mit geringerer Mühe und weniger Zeitaufwand anzufertigen, als ein ausgestopfter Balg.

Reisenden, Jägern und Anderen, die nicht schöne Sammlungen anlegen wollen, sei das e infachere Verfahren empfohlen, das darin besteht, das Präparat nach oberflächlichem oder durchgreifendem Trocknen zu verpacken und zu versenden. Will der Sammler ein erbeutetes Thier sofort nach seinem Bestimmunsgorte transportiren, der innerbalb weniger Tage zu erreichen ist, so ist es am einfachsten, das Objekt nur zu injiciren, mit oder ohne den Darm. Anstatt zu injiciren kann man aber noch anders verfahren. Man präparire sich zu dem Zweck Watte oder auch Wolle, die man mit Sublimatlösung, Carbolsäure oder dergl. vergiftet, und verfertige davon kleine Nudeln, welche man nach dem Trocknen in den Darm, in den Rachen etc. schiebt. Dies kann jeder Laie mit Leichtigkeit ausführen, zumal im Nothfall die in den Apotheken käufliche Carbol- oder Salicylwatte ausreichend ist. In einem solchen Zustande wird sich das Object so lange halten, bis es an Ort und Stelle angelangt, endgültig präparirt werden kann.

Forschungsreisenden, welche sich mit dem Abbalgen nicht aufhalten wollen, empfiehlt es sich, die äussere Vergiftung vorzunehmen. Darin liegt ja gerade ein grosser Vortheil von dem bisherigen Verfahren, da hierbei selten der Balg vergiftet wird und daher zu leicht dem Verderben ausgesetzt ist, denn durch das Ausschmieren desselben mit Arsenik wird das Ge fie der nicht vergiftet. Es wird daher auch rathsam sein, den abgezogenen Balg in der oben angegebenen Weise mit Sublimat zu vergiften, 
indem man ihn darin badet oder damit benetzt und dann trocknet, welch' letzteres übrigens auch schneller als sonst geschehen kann, da durch den Spiritus der Haut ein Theil ihres Wassergehaltes entzogen wird. -

Aus dem Bisherigen lässt sich anderseits aber auch errathen, dass die Anwendbarkeit des Mumificirens entweder keine ganz allgemeine oder doch nicht überall angebrachte ist. Grössere Objecte, wie etwa Adler und Enten trocknen zu schwer aus. Ausserdem nehmen sie beim Verpacken mehr Platz in Anspruch, als der leere Balg. Da es in vielen Fällen aber nicht erwünscht ist, ausser dem Balg noch das Skelett zu besitzen, und zwar unbeschädigt, so möchte ich vorschlagen, ausser der Bauchhaut mit demselben Längeschnitt noch die Brusthaut zu öffnen und die grössere Masse der Brustmusculatur zu entfernen, die gerade das Massigte ist und am schwersten austrocknet.

Die meisten der bedeutenderen Museen besitzen. Sammlungen von Vogelbälgen, die unmöglich alle ausgestopft und ausgestellt werden können. Dies artet auch gar zu leicht in handwerksmässige Spielerei aus. Man begnügt sich daher damit, den Balg als solchen zu konserviren, welcher für den rein wissenschaftlichen Zweck ja dasselbe leistet wir der armirte Vogel. Diesen selben Zweck erfüllen aber auch unsere Mumien, sei es, dass man sie noch nachträglich abbalgt. Es ist hiernach auch selbstverständlich, dass sie auch noch ausgestopft werden können, wozu sie sich in mancher Beziehung besser eignen, als ein alter Balg, da die Haut viel gleichmässiger getrocknet ist und sich nicht so verziehen konnte, wie dies oft genug bei den Bälgen eintritt, so dass diese nachher beim Ausstopfen schwer in die richtige Form zu bringen sind.

Zum Schluss möge noch darauf hingedeutet werden, dass sich das Mumificationsverfahren auch auf viele andere zoologische Objecte anwenden lässt, wenn man nichts Besseres zur Hand hat. Nur in wenigen Fällen, wie etwa bei den Insecten oder Echinodermen, wird man dabei ohne Weiteres gute Museumspräparate erzielen können. Häufig handelt sich es aber nur darum, ein Thier näher zu bestimmen, damit der daran Interessirte weiss, womit er es zu thun hat, und dann empfiehlt es sich immer, das Object $\mathrm{zu}$ vergiften und zu trocknen, um es an ein Museum oder einen Fachmann zu senden, welcher die Determination vornehmen kann. Es ist dann nur nöthig, das Präparat in Alcohol oder sonstwie aufzuweichen, wenn es schon zu hart ist. 
Nach einer sorgfältigen Behandlung mit Sublimatalcohol kann man endlich eine ganze Reihe von Thieren trocken conserviren, wenn man hierbei einige Kunstgriffe anwendet. Das erste Erforderniss hierzu ist ein langes, womöglich mehrwöchiges Verweilen derselben in dieser Flüssigkeit. Werden sie dann herausgenommen, so lasse man sie nicht schnell austrocknen, sondern bedecke sie, um dies zu verhindern, mit einem Kasten oder dergl. Gleichzeitig stopfe man durch die Körperöffnungen Watte und ähnliche Stoffe nach, um die Haut immer prall zu erhalten. Dies lässt sich mit Glïck allerdings nur bei Thieren mit fester, harter Körperbedeckung ausführen, wie bei Schlangen, Eidechsen und einer Anzahl von Fischen. Andere Objekte, wie Frösche kann man aufblasen, oder besser noch, mit trocknem Sande ausfüllen, den man nach dem Trocknen wieder ausschïttet. Ein sehr geeignetes Füllmaterial geben ferner Flüssigkeiten ab, die später erhärten, wie etwa eine vergiftete Leimabkochung, Paraffin, Fett etc., die man mittels einer Spritze in die Leibeshöhle einführt. Wo Gliedmaassen vorhanden sind, wie bei Säugern. Fröschen etc. ist es gut, an passender Stelle eine Oeffnung herzustellen, um von dort aus das Material einzuführen.

Ein derartiges Präparationsverfahren eignet sich namentlich für kleinere Thiere, deren Abbalgen nicht ganz leicht und deren Ausstopfen schwierig ist, wie kleine Mäuse, Salamander, Eidechsen, Blindschleichen etc. Die so hergestellten Präparate haben weniger einen wissenschaftlichen, als vielmehr einen pädagogischen Zweck und können den Anschauungsunterricht mehr fördern, als in Gläsern und Alcohol eingeschlossene, und bei geschickter Behandlung bewahren sie ihre natürlichen Formen besser, als ausgestopfte Bälge, die zumeist etwas Gekünsteltes an sich haben.

\section{A. B. Meyer, Abbildungen von Vogel-Skeletten,}

herausgegeben mit Unterstütung der Generaldirection der K. Sammlungen für Kunst und Wissenschaft in Dresden. Band I. Mit $121 \mathrm{~T}$ afeln (I-CXX) in Lichtdruck. Dresden 1879-88.

\section{Bericht von M. Fürbringer.}

Die 12. und 13. Lieferung von A. B. Meyer's Abbildungen von Vogelskeletten ist am Anfange dieses Jahres*) edirt worden.

*) Das Manuscript wurde 1890 eingesandt. 


\section{$2 \mathrm{BHL}$ Biodiversity Heritage Library}

1891. "Verfahren zur Mumification von Vögeln und anderen zoologischen Objecten." Journal

$f u$

r Ornithologie 39, 74-86. https://doi.org/10.1007/bf02252222.

View This Item Online: https://www.biodiversitylibrary.org/item/101710

DOI: https://doi.org/10.1007/bf02252222

Permalink: https://www.biodiversitylibrary.org/partpdf/142943

\section{Holding Institution}

Smithsonian Libraries

\section{Sponsored by}

Biodiversity Heritage Library

\section{Copyright \& Reuse}

Copyright Status: Public domain. The BHL considers that this work is no longer under copyright protection.

This document was created from content at the Biodiversity Heritage Library, the world's largest open access digital library for biodiversity literature and archives. Visit BHL at https://www.biodiversitylibrary.org. 